\title{
Fat necrosis of the breast - report of three cases
}

\section{Peter Evan, MUDR (Prague), Dip Rad \\ Candy Pather, MB ChB, FC Rad (Diag)}

Department of Radiology, Netcare Park Lane Clinic, Parktown, Johannesburg

\section{Abstract}

Fat necrosis is a common benign condition. It is associated with iatrogenic, penetrating or blunt trauma. In a number of cases, there is no history of injury. Mammographic presentation is either a typical lipid cyst or a spiculated lesion, mimicking malignancy. Ultrasonographic appearance is that of either a cyst, or solid mass, or an area of increased echogenicity. Calcifications may be present and are usually coarse or ring-like.

\section{Introduction}

Fat necrosis (FN) is a benign condition, occurring as a result of trauma. It has a variety of clinical, mammographic and ultrasonographic appearances. We present three illustrative cases, one after previous lumpectomy, one after breast augmentation and one with no history of trauma or surgery.

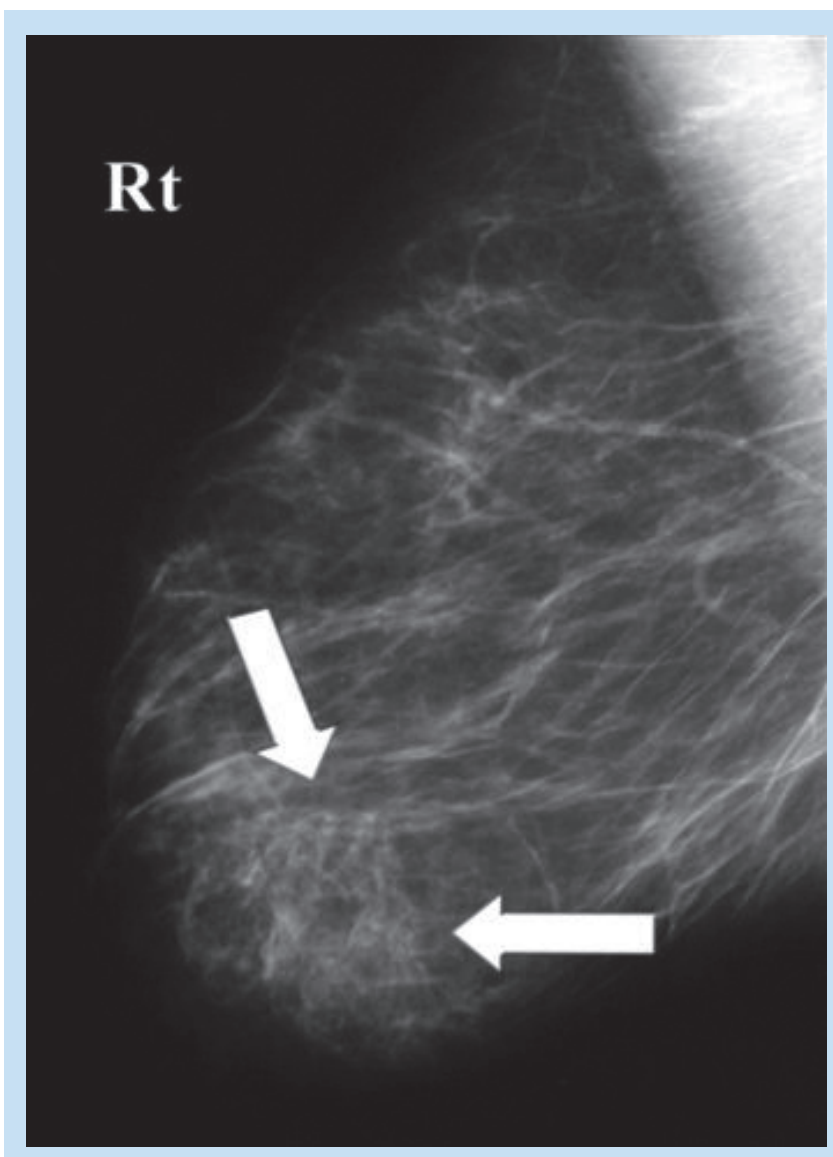

Fig. 1. MG. Area of increased density in the lower medial quadrant of the right breast near the areola. Distortion of the glandular architecture.

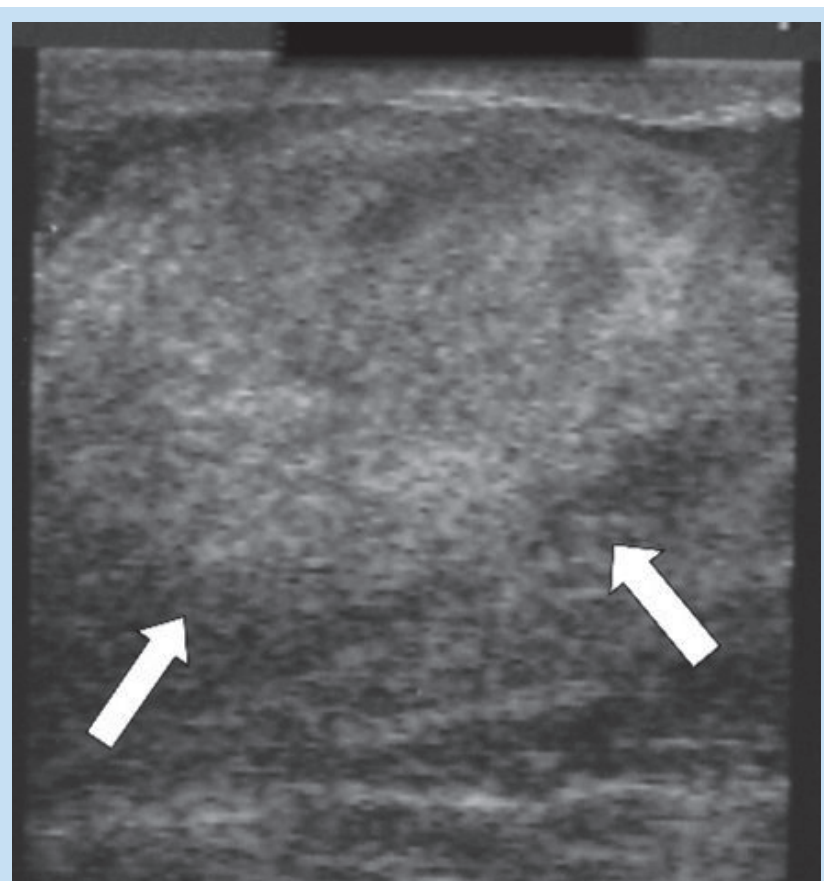

Fig. 2. US. Well-outlined area of increased echogenicity. No spiculae. No calcifications. No increased flow on colour Doppler.

\section{Cases}

Case 1 (Figs 1 \& 2)

A week previously a 56-year-old woman discovered a hard lump in her right breast. A palpable mass $3 \times 4 \mathrm{~cm}$ in the lower/medial quadrant of the right breast was found. It was movable with no skin thickening. An area of increased density with architectural distortion was seen on mammography (MG). There were no microcalcifications (Fig. 1). On ultrasonography (US), there was a well-outlined area of increased echogenicity (Fig. 2). No increased flow was seen on duplex Doppler examination. She had no history of surgery or trauma. Core biopsy was done and reported as fat necrosis. The patient was given reassurance. No further treatment was necessary.

\section{Case 2 (Figs 3 \& 4)}

A 48-year-old woman had had a benign lump removed from her left breast 6 years previously. She had a tender, palpable mass at the site of previous surgery. MG demonstrated a dense, spiculated lesion in the lower lateral quadrant of the left breast (Fig. 3), corresponding to the region of the scar. There were no calcifications and no translucent centre. On US, the lesion was seen as a circumscribed area of decreased echogenicity, measuring $20 \times 8 \mathrm{~mm}$, orientated with the long axis parallel to the skin (Fig. 4). Core biopsy was reported as post-traumatic fat necrosis.

\section{Case 3 (Fig. 5)}

This 55-year-old woman was referred for MG (in 1991) for a hard mass in each breast. MG demonstrated a well-outlined, calcified mass in each breast. It measured about $5 \mathrm{~cm}$ in diameter. The mass on the right had 


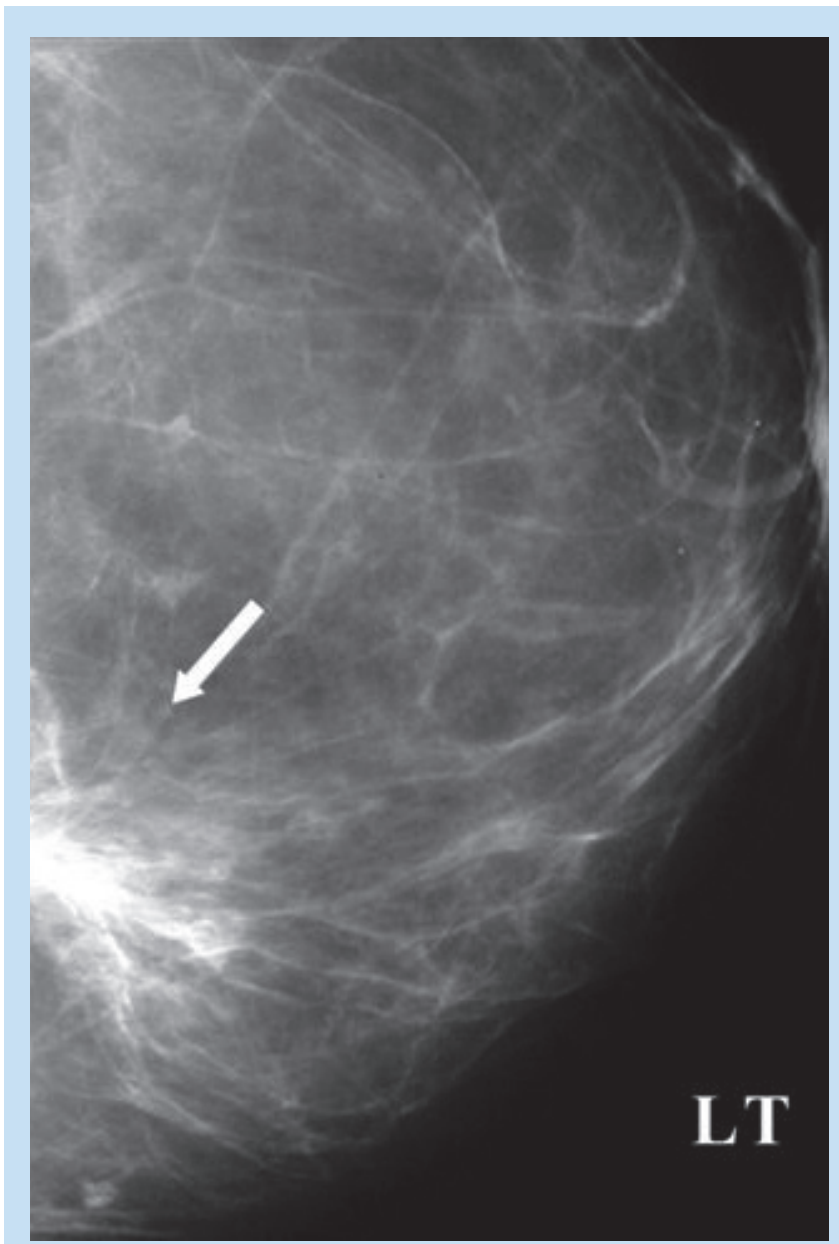

Fig. 3. MG. Spiculated mass in the lower lateral quadrant left breast (arrow). No translucent centre. No calcifications.

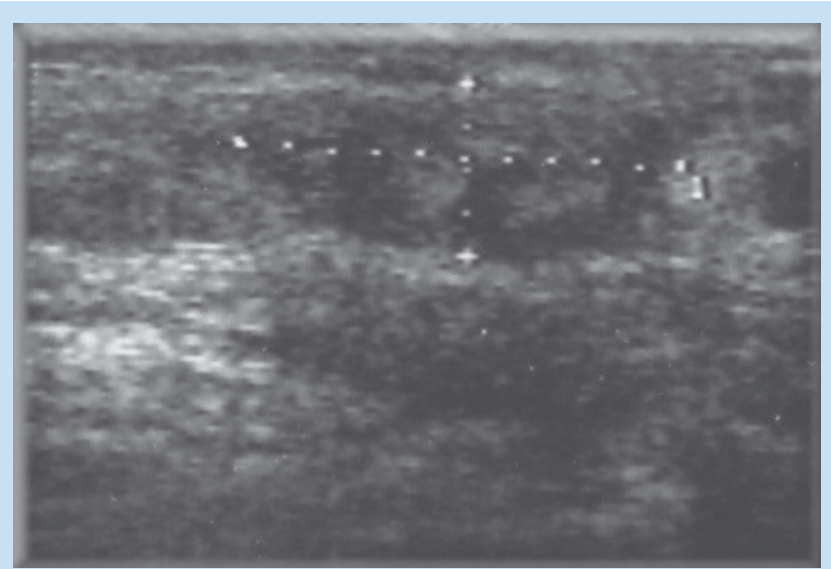

Fig. 4. US. Circumscribed mass $20 \times 8 \mathrm{~mm}$. Long axis parallel to the skin. No calcifications. No spiculae. No increased flow.

a calcified rim. In addition, multiple small, cyst-like calcifications were noted throughout both breasts. She had had breast augmentation 23 years previously. Fat taken from her own buttocks was used instead of a prosthesis. She was asymptomatic and happy with the results. The diagnosis of FN was obvious and biopsy was not done.

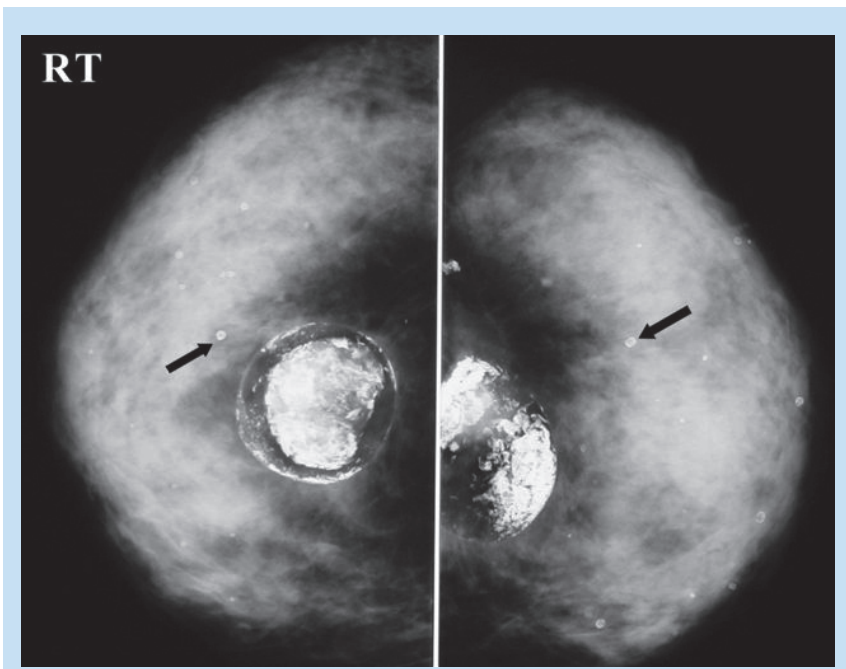

Fig. 5. Symmetrical mass in each breast with calcified centre and calcified rim. Multiple small round calcifications with translucent centre, typical of oil cysts (arrows).

\section{Discussion}

FN was first described in 1920 by Lee and Munzer (quoted by Hogge et al. $\left.{ }^{1}\right)$. History of trauma was noted in $40 \%$ of cases. It was thought to occur in the superficial breast tissue and sub-areolar region in obese patients with pendulous breasts. ${ }^{2}$ However, men can also be affected. Causes are listed as iatrogenic and non-iatrogenic.

\section{Iatrogenic causes}

These include biopsy, aspiration, lumpectomy and any other breast surgery. Reconstructive surgery such as transverse rectus abdominis musculocutaneous (TRAM) flap, autogenous myocutaneous flap and breast reduction has an incidence of FN in up to $25 \%$ of cases. ${ }^{4}$ Radiation therapy ${ }^{1,3,5}$ is also implicated. Salhab et al. ${ }^{6}$ reported FN after injection of methylene blue dye for sentinel node biopsy. Patent blue $\mathrm{V}$ should be used instead. FN can be the result of anticoagulation therapy ${ }^{7}$ with both unfractioned and low-molecular-weight heparin.

\section{Non-iatrogenic causes}

FN is seen after either penetrating trauma, such as stab wounds, gun shots, dog bite and other accidents, ${ }^{3}$ or blunt trauma due to a direct hit to the anterior chest wall. In motor-car accidents, the impact of the steering wheel may cause FN. Seat-belt injuries to the breast have been described by DiPiro et al. ${ }^{8}$ On MG and US they present in two forms: $(i)$ band-like areas of increased density compatible with haematoma and/or contusion; and (ii) nodular parenchyma. With time, these signs decrease in prominence. Linear distribution of thin-walled oil cysts is typical and was seen in all patients. The cysts either resolve or calcify. The location of the injury correlates well with the position of the seat belt. We can expect to see more cases of FN in young women, as they participate in contact sports such as rugby, soccer and boxing. A large number of patients have no history of trauma and may have had a minor accident that was long forgotten.

\section{Clinical presentation}

The patient is often asymptomatic ${ }^{1,3,9,10}$ and FN is discovered as an inci- 
dental finding during screening MG, or at post-surgery follow-up.

The most common symptom is a palpable mass, which may be irregular or fixed. There is often pain and tenderness. Multiple smooth nodules are occasionally seen. Skin thickening, induration, ecchymosis and occasionally lymphadenopathy are present. As the $\mathrm{FN}$ is often situated near the areola, retraction of the nipple and/or areola may be found.

The incidence of FN was 2\% in 536 breast biopsies reported by Hogge et al. ${ }^{1}$ The incidence is higher in patients after surgery. Dershaw et al. ${ }^{11}$ report on 28 women with suspected recurrence after lumpectomy for malignancy. Twenty-nine biopsies were performed. Three patients had microcalcifications. Of the 26 palpable masses, 10 masses were malignant, 3 of them not demonstrated on MG. Of the 16 benign masses, 7 were not visible on MG. The histological findings included fibrosis in 8, FN in 7 and granuloma in 1 case. The palpable mass may enlarge slowly over a few weeks and may or may not undergo spontaneous regression. Diligent clinical examination is important, as a palpable mass was not demonstrated on MG in $9.5 \%$ and on US in $10 \%$.

\section{Mammographic findings}

Lipid (oil) cyst. Presents as a round or oval, lucent mass with smooth borders. ${ }^{1}$ The rim of the cyst may calcify. The appearance is benign in most cases. Early calcification or collapse of the fibrous rim may produce indeterminate appearance requiring biopsy. ${ }^{1}$

Focal mass. A common finding is a dense focal mass with or without calcifications (Fig.1). Distortion of the glandular architecture is often seen. This is a subtle sign and must be searched for. The mass may be totally or partially calcified (Fig. 5).

Spiculated area of increased density. This occurs when fibrosis predominates (Figs $1 \& 3$ ) and produces desmoplastic reaction mimicking scirrhous carcinoma. ${ }^{1}$

Microcalcifications. Focally clustered pleomorphic calcifications are rare. They may have rod-like or angular configuration ${ }^{13}$ and are indistinguishable from those of carcinoma. Coarse and homogenous calcifications are common and are indicative of a benign lesion (Fig. 5).

\section{Ultrasound features}

There is a large variety of US appearances. Most commonly a solid mass, usually well circumscribed and of low echogenicity, is seen (Fig.4). It may have either posterior acoustic shadowing or enhancement or neither. When cystic, the cyst may be septated, complex or with a mural nodule. The cysts may contain oil-fluid levels. ${ }^{12}$ Often, the only US sign is an area of increased echogenicity in the subcutaneous region (Fig. 2).

\section{Pathology}

FN is a benign, non-suppurative inflammatory process. ${ }^{9}$ It is the result of aseptic saponification of the fat by blood and tissue lipase. It contains anuclear fat cells, surrounded by giant histiocytes and aggregates of foamy phagocytic histiocytes. Fibrotic connective tissue is seen at the periphery of the necrotic debris. The extent of fibrotic reaction correlates well with MG finding of a dense, spiculated lesion.

\section{Conclusion}

FN is a benign condition, usually the result of trauma, most commonly iatrogenic. The appearances are either typical, in which case no further evaluation is needed, or may mimic malignancy which would require biopsy to distinguish them from recurring carcinoma.

1. Hogge JP, Robinsom RE, Magnant CM, Zuurbier RA. The mammographic spectrum of fat necrosis of the breast. RadioGraphics 1995: 15; 1347-1356.

2. Haagensen CD. Disease of the Breast, 3rd ed. Philadelphia: Saunders, 1986: 369-378.

3. Jackson VP, Fu KL, Fu YS. Benign breast lesions. In: Bassett LW, Jackson VP, Fu KL, Fu YS, ed. Diagnosis of Diseases of the Breast. 2nd ed. Philadelphia: Elsevier Saunders, 2005: 409-419.

4. Hogge JP, Zuurbier RA, de Paredes ES. Mammography of autologous myocutaneous flaps. RadioGraphics 1999; 19: S63-S72.

5. Kim SM, Park JM. Mammographic and ultrasonographgic features after autogenous myocutaneous flap reconstruction mammoplasty. J Ultrasound Med 2004; 23: 275-282.

6. Salhab M, Al Sarakbi W, Mokbel K. Skin and fat necrosis of the breast following methylene blue dye injection for sentinel node biopsy in a patient with breast cancer. Semin Surg Oncol 2005; 2: 26.

7. Das AK. Low molecular-weight heparin-associated fat necrosis of the breast. Age Ageing 2005; 34: 193 194.

8. DiPiro PJ, Meyer JE, Frenna TH, Denison CM. Seat belt injuries of the breast. AJR 1995; 164: 317-320.

9. Bassett LW, Gold RH, Cove HC. Mammographic spectrum of traumatic fat necrosis: the fallibility of "pathognomonic" signs of carcinoma. AJR 1978; 130: 119-122.

10. Bilgen IG, Ustun EE, Memis A. Fat necrosis of the breast: clinical, mammographic and sonographic features. Eur J Radiol 2001: 39: 92-99.

11. Dershaw DD, McCormick B, Cox L, Osborne MP. Differentiation of benign and malignant tumor recurrence after lumpectomy. AJR 1990; 155: 35-38.

12. Kim HS, Cha ES, Kim HH, Yoo JY. Spectrum of sonographic findings in superficial breast masses. J Ultrasound Med 2005; 24: 663-680.

13. Bassett LW, Gold RH, Mirra JM. Nonneoplastic breast calcifications in lipid cysts: development after excision and primary irradiation. AJR 1982: 138: 335-338 